\title{
Pulse shortening by a nonlinear mirror mode locker
}

\author{
Krassimir Stankov
}

\begin{abstract}
A device, composed of a nonlinear crystal and a dichroic mirror, features intensity-dependent reflection and may be used as a passive mode locker. The pulse shortening of a Gaussian light pulse due to reflection by this nonlinear mirror has been calculated and compared to that of a saturable absorber used for mode locking.
\end{abstract}

\section{Introduction}

Saturable absorbers exhibit intensity-dependent light transmission. In mode-locked lasers this feature is used to perform amplitude discrimination and pulse shortening. Pulse shortening has been analyzed in the case of a fast absorber, ${ }^{1}$ in the intermediate case, ${ }^{2}$ and has been calculated for an arbitrary ratio of the pulse duration to the absorber relaxation time. ${ }^{3,4}$

Recently a novel nonlinear optical device utilizing second harmonic generation was proposed. ${ }^{5}$ It may exhibit intensity-dependent reflection or transmission with a fast time response in the subpicosecond range. Successful mode locking of a Nd:YAG laser using this device was demonstrated. ${ }^{6,7}$ Since one of the important characteristics of a mode locker is pulse shortening in a single transit, in this paper we calculate pulse shortening due to reflection by the nonlinear mirror and make a comparison to the performance of a saturable absorber used in a mode-locked laser.

\section{Pulse Shortening for a Normalized Gaussian Pulse}

The nonlinear mirror is a combination of a nonlinear crystal generating second harmonic and a dichroic mirror placed behind it (Fig. 1). The incident light generates second harmonic in the crystal and the dichroic mirror reflects totally the second harmonic and partially the fundamental radiation back into the nonlinear medium. Some means are provided to adjust the phase difference $\varphi=2 \varphi_{1}-\varphi_{2}\left(\varphi_{1}, \varphi_{2}\right.$ are the phases of the fundamental and second harmonic waves) to be $-\pi / 2+2 m \pi$. After reflection by the dichroic mirror in

The author is with Max-Planck-Institute für biophysikalische Chemie, Abteilung Laserphysik, P.O. Box 2841, D-3400 Göttingen, Federal Republic of Germany.

Received 10 March 1988.

0003-6935/89/050942-04\$02.00/0.

(C) 1989 Optical Society of America. the second pass through the crystal a reconversion of the second harmonic into fundamental takes place. The reflection of this device in these conditions increases by increasing the light intensity.

If the phase difference is $\varphi=\pi / 2+2 m \pi$, in the second pass further conversion into second harmonic is provided. Then, the action of the device is equivalent to a double-pass second harmonic generation with some modification of the wave intensities after the first pass. In this case the reflected intensity decreases by increasing the input one.

The analysis ${ }^{5}$ shows that both higher conversion efficiency and lower reflectivity of the dichroic mirror at the fundamental wavelength enhance the nonlinearity in light reflection. An expression for the nonlinear reflection coefficient at the fundamental wavelength $R_{N L}$ as a function of the conversion efficiency $\eta$ and the dichroic mirror reflectivities at the fundamental, $R 1$, and the second harmonic, $R 2$, has been derived for the case of interest, i.e., when $\varphi=-\pi / 2+2 m \pi$. If the dichroic mirror is totally reflecting at the second harmonic, the nonlinear reflection is given by

$$
R_{N L}=B\left\{1-\tanh ^{2}[\sqrt{B} \cdot \operatorname{arctanh} \sqrt{\eta}-\operatorname{arctanh}(\sqrt{\eta / B})]\right\},
$$

where

$$
B=\eta+(1-\eta) R 1 .
$$

The reflection coefficient $R 1$ may be regarded as a small-signal reflection analogous to the small-signal transmission of a saturable absorber. If we consider a light pulse reflected by the nonlinear mirror, the highintensity parts of the pulse will be more efficiently reflected than the low-intensity parts. Hence, one expects pulse shortening, similar to that experienced by a light pulse passing through a saturable absorber. In accordance with the above brief discussion on the effect of the phase difference it is evident that the pulse shortening maximizes for $\varphi=-\pi / 2+2 m \pi$. Deviation from this will reduce the pulse shortening and approaching $\varphi=\pi / 2+2 m \pi$ pulse broadening will result. 


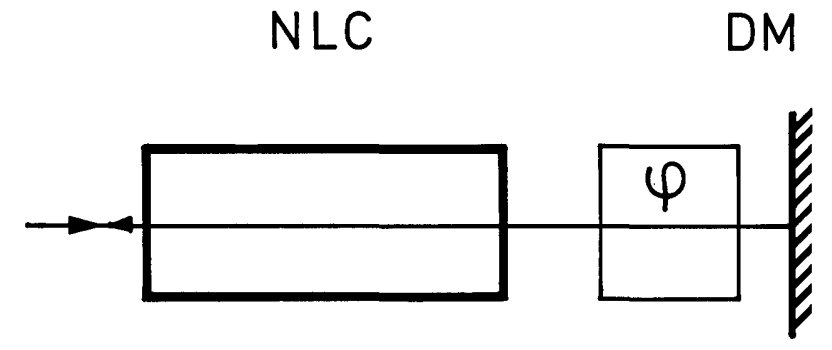

Fig. 1. Nonlinear mirror: $N L C$, nonlinear crystal for second harmonic generation; $\varphi$, phase adjustment; $D M$, dichroic mirror (total reflector at the second harmonic wavelength, partial reflector at the fundamental).

In what follows, we calculate the pulse shortening of a normalized Gaussian pulse with FWHM $=1$ for $\varphi=$ $-\pi / 2+2 m \pi$. A phase difference change within $+/$ $-(\pi / 6)$ reduces the mode-locking probability to $\sim 50 \%$ (see Fig. 3 in Ref. 7).

As in Ref. 5, we confine our treatment to plane waves and perfect phase matching. The walk-off effect is compensated, since after reflection by the dichroic mirror the fundamental and second harmonic beams travel exactly the same optical paths. Thus, the only influence of the walk-off effect is reduction of the conversion efficiency. In addition, we assume pulse durations $\tau_{p}$ for which the crystal length is smaller than $L_{\tau}=\tau_{p} /\left(u_{1}^{-1}-u_{2}^{-1}\right)^{-1}$, where $u_{1,2}$ are the group velocities for the fundamental and the second harmonic. This assures that the group velocity mismatch does not broaden the second harmonic pulse. ${ }^{8}$ For reasonably high conversion efficiencies the pulse durations in these conditions are in the $0.2-5$-ps range, depending on the type of crystal and the wavelength region. Higher-order effects like dispersion spreading are neglected.

First we analyze the pulse shortening as a function of the peak conversion efficiency. Thus, the incident Gaussian pulse with FWHM = 1 has the form

$$
I(t)=\exp \left(-4 \cdot \ln 2 \cdot t^{2}\right) .
$$

We use the following procedure: the instantaneous conversion efficiency $\eta(t)$ is determined using the relation

$$
\eta(t)=\tanh ^{2}\left[\sqrt{I(t)} \cdot \operatorname{arctanh} \sqrt{\eta_{0}}\right],
$$

where $\eta_{0}$ is the peak conversion efficiency at $t=0$ for a pulse of unity peak intensity. Next, the instantaneous nonlinear reflection coefficient $R_{N L}(t)$ is calculated, substituting Eq. (4) into Eqs. (1) and (2). The reflected pulse form $I_{r}(t)$ (at the exit of the nonlinear mirror, i.e., at the left-hand side of the crystal $N L C$ ) is given by

$$
I_{r}(t)=I(t) \cdot R_{N L}(t) .
$$

Lastly, we calculate the time at which the reflected intensity drops to half of its maximum value. Figure 2 illustrates the results of these calculations. The full width at half-maximum $(\tau)$ of the reflected pulse is plotted as a function of the peak conversion efficiency of the incident pulse for various values of the dichroic

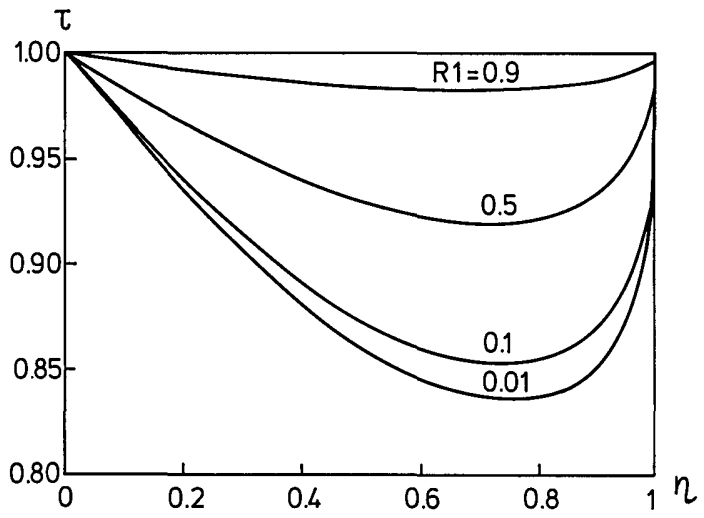

Fig. 2. Pulse width $\tau$ after reflection of a normalized Gaussian pulse with FWHM $=1$ as a function of the peak conversion efficiency $\eta$ for various small-signal reflection coefficients $R 1$.

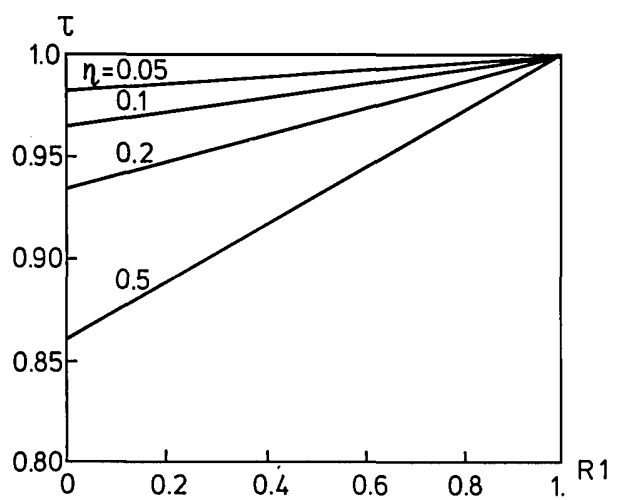

Fig. 3. Pulse width dependence on the small-signal reflectivity $R 1$ for various conversion efficiencies $\eta$.

mirror reflectivity $R 1$ at the fundamental wavelength. As one can expect, the pulse shortening is more pronounced for high conversion efficiency. However, it peaks at a conversion efficiency in the range of $0.6-0.8$ and then rapidly approaches the incident pulse duration. This is to be expected, since for a very high conversion the second harmonic pulse follows closely the pulse form of the fundamental (of course not in the wings) due to saturation.

By inspection of the expression for the reflected pulse form [Eq. (5)] it can be seen that the pulse shape is modified by the nonlinear reflection coefficient, which is a rather complex function. Thus, the reflected pulse shape does not follow the input one (Gaussian or $\operatorname{sech}^{2}$ ).

From Fig. 2 it is also evident that the pulse shortening is higher for lower reflection coefficient $R 1$. This is in accordance with the features of the nonlinear mirror mentioned above. The dependence of the pulse shortening on the dichroic mirror reflectivity $R 1$ for various peak conversion efficiencies is presented in Fig. 3 in detail. Here we remind the reader that the pulse shortening and the amplitude discrimination starts at a conversion efficiency of the order of several percent (the upper most curve in Fig. 3) as the mode-locking experiment $t^{6,7}$ has revealed. A reasonable question is 
$\tau$

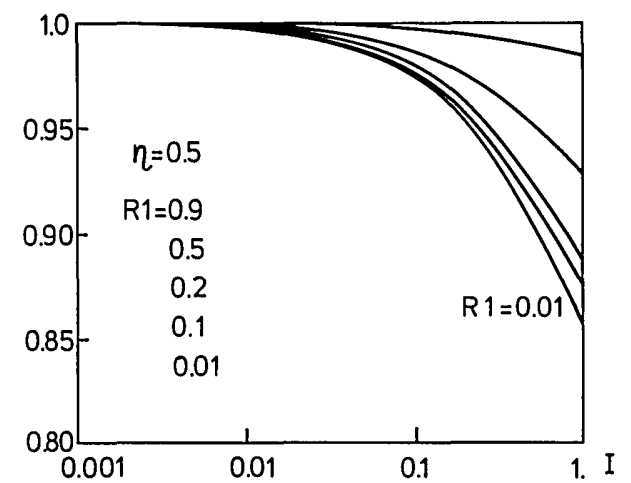

Fig. 4. Pulse width as a function of the incident peak intensity for different small-signal reflections $R 1$. For incident unity peak intensity conversion efficiency of 0.5 is assumed. The upper most curve is relevant to $R 1=0.9$, the lowest one to $R 1=0.01$.

why is the pulse shortening not close to $1 / \sqrt{2}$, as one can expect for low conversion efficiency. It is well known that for a Gaussian pulse after the first pass through the nonlinear crystal the second harmonic pulse is shortened with respect to the fundamental by this factor. The reason is that the fundamental pulse will broaden because of the depletion of the high intensity parts. In the second pass through the crystal the broadened fundamental pulse is amplified in the field of the second harmonic pulse, thus the effect of the pulse shortening in the second harmonic generation is somewhat reduced.

Finally, we illustrate pulse shortening as a function of the incident peak pulse intensity. Conversion efficiency of 0.5 is assumed for a pulse having unity peak intensity (Fig. 4).

\section{Discussion}

This part of the paper is devoted to a comparison of pulse shortening by the nonlinear mirror to that of a saturable absorber when used as a mode locker. The advantages of the nonlinear mirror such as simplicity, reliability, wide spectral ranges of operation, simultaneous generation of ultrashort light pulses at the second harmonic, and fast time response have been discussed in Refs. 5-7. Because of the fast time response one might expect better performance of the nonlinear mirror in comparison with slow absorbers, at least for solid-state lasers. ${ }^{9}$ Therefore we compare pulse shortening to that of a fast absorber. When a single-pass extracavity pulse shortening is required, ${ }^{4}$ saturable absorbers of very low small-signal transmission of the order of $10^{-7}$ are superior and may provide an optimized pulse reduction by a factor of 3 . However, in mode-locked solid-state lasers, ${ }^{10}$ the small-signal transmission ranges from 0.5 to 0.8 . The optical arrangement with an absorber corresponding to the nonlinear mirror is a dye cell with a totally reflecting mirror behind it. Since the light passes twice through the cell, the low intensity transmission is $0.25-0.64$. Thus, the corresponding relative pulse shortening [defined as $\left.\left(\tau-\tau_{p}\right) / \tau_{p}\right]$ may reach values in the range from
-0.16 to $-0.0 \overline{6}$ (see Fig. 2 in Ref. 1). Here we note that these are the maximum values attainable at an optimized intensity level only.

Considering a nonlinear mirror, intracavity conversion efficiencies of 0.5 can be realized with highly efficient nonlinear crystals such as KTP and BBO. For $R 1=0.25-0.64$ (small-signal reflection) the relative pulse shortening (see Fig. 4 ) is in the range from -0.11 to -0.05 , i.e., somewhat smaller but very close to that of the absorber. However, in a mode-locked laser the absorber pulse shortening maximizes at a certain intensity level, while the nonlinear mirror one increases monotonically for all realistic values of intensities and conversion efficiencies.

To reveal the ultimate capabilities of the nonlinear mirror with respect to pulse shortening one has to perform an analysis assuming nonstationary second harmonic generation and reconversion. ${ }^{8}$ However, some crude estimates are possible. For the experiment ${ }^{6,7}$ where $5.5-\mathrm{mm}$ long KTP crystal was used, effective pulse shortening may be expected for pulses as short as 4 ps (estimated from the data in Ref. 11). Especially favorable is the situation for mode locking a Nd:glass laser using a KDP crystal. In this case for a 2cm long crystal pulses as short as $200 \mathrm{fs}$ may still undergo pulse shortening. ${ }^{12}$ In both examples one can expect generation of ultrashort pulses with a duration determined by the spectral gain profile (if there are no other limitations, such as self-phase modulation or limited number of round trips). A comprehensive analysis of laser dynamics, including the effect of the nonlinear mirror and laser active medium, is out of the scope of this paper.

It is worth mentioning that the change of phase difference $\varphi$ does not limit the bandwidth of the device, but only changes the direction of the interaction between the lightwaves in the second pass, i.e., reconversion back into fundamental or further conversion into second harmonic. Also, since the group velocity dispersion in air is several orders of magnitude less than in nonlinear crystals, ${ }^{13}$ the crystal mirror separation does not impose any practical limitations on the performance of the nonlinear mirror mode locker with respect to the bandwidth.

\section{Conclusion}

We have presented an analysis of pulse shortening due to reflection by a nonlinear mirror. In this respect the performance of the nonlinear is comparable to that of a saturable absorber mode locker. However, due to a number of other advantages one can expect that in many cases the new mode-locking device will replace the saturable absorbers in the passive mode-locking technique. Using other nonlinear processes such as sum or difference frequency generation ${ }^{14}$ or Raman scattering, this technique may be extended to all wavelength regions for which suitable nonlinear media exist.

Useful discussions with F. P. Schafer and G. Marowsky and valuable comments of G. Szabo are grate- 
fully acknowledged. The author holds a research fellowship from the Alexander von Humboldt Foundation, on leave from Department of Physics, Sofia University, Bulgaria.

\section{References}

1. W. Krause, "A Pulse Compression Analysis for the Fast Saturable Absorber of Arbitrary Small-Signal Transmittance," Opt. Commun. 48, 47 (1983).

2. W. Rudolf and H. Weber, "Analysis of Saturable Absorbers, Interacting with Gaussian Pulses," Opt. Commun. 34, 491 (1980).

3. A. Penzkofer, D. von der Linde, A. Laubereau, and W. Kaiser, "Generation of Single Picosecond and Subpicosecond Light Pulses," Appl. Phys. Lett. 20, 351 (1972).

4. A. Penzkofer, "Generation of Picosecond and Subpicosecond Light Pulses with Saturable Absorbers," Opto-electronics 6, 87 (1974).

5. K. A. Stankov, "A Mirror with an Intensity-Dependent Reflection Coefficient," Appl. Phys. B 45, 191 (1988).

6. K. A. Stankov, "A Novel Nonlinear Optical Device for Passive Mode-Locking," in Technical Digest, Conference on Lasers and
Electro-Optics (Optical Society of America, Washington, DC, 1988), paper THJ4.

7. K. A. Stankov and J. Jethwa, "A New Mode-Locking Technique Using a Nonlinear Mirror," Opt. Commun. 66, 41 (1988).

8. S. A. Akhmanov, A. S. Chirkin, K. N. Drabovich, A. I. Kovrigin, R. V. Khokhlov, and A. P. Sukhorukov, "Nonstationary Nonlinear Optical Effects and Ultrashort Light Pulse formation," IEEE J. Quantum Electron. QE-4, 598 (1968).

9. E. G. Arthurs, D. J. Bradley, and T. J. Glynn, "The Effect of Saturable Absorber Lifetime in Picosecond Pulse Generation. I. The Ruby Laser," Opt. Commun. 12, 136 (1974).

10. J. Herrmann and B. Wilhelmi, Laser für ultrakurze Lichtimpulse (Akademie-Verlag, Berlin, 1984).

11. R. F. Belt, G. Gashurov, and Y. S. Liu, "KTP as a Harmonic Generator for Nd:YAG Lasers," Laser Focus/E-O, 110 (Oct. 1985).

12. J. Comly and E. Garmire, "Second Harmonic Generation from Short Pulses," Appl. Phys. Lett. 12, 7 (1968).

13. A. Brimontas, V. Vasilyauskas, A. Piskarskas, and A. Stabinis, "Dispersion Spreading of Femtosecond Light Pulses in Crystals, Air and Water," Sov. J. Quantum Eletron. 15, 787 (1985).

14. K. A. Stankov, "New Methods of Passive and Active ModeLocking Using Intracavity Optical Frequency Mixing," Opt. Lett. accepted.

\section{OPTICAL ENGINEERING SHORT COURSES}

Attendees may choose four courses from the following eight, which will be offered from May 8 - 19, 1989, at the Doubletree Hotel in Tucson, Arizona.

May $8-10$
Geometrical Optics
and Lens Design
Roland V. Shack
Robert R. Shannon
George N. Lawrence
Optical Coatings
H. Angus Macleod
Michael R. Jacobson

May $11-12$
Principles of Fourier
Optics and Holography
Jack D. Gaskill
Raymond K. Kostuk
Scattered Light in
Optical Systems
William L. Wolfe
Robert P. Breault

May $15-17$

Opto-mechanical Design

and Optical Alignment

Daniel Vukobratovich

Mitchell C. Ruda

Optical Radiation Detectors

William L. Wolfe

Eustace L. Dereniak

James M. Palmer
May $18-19$

Optical testing

James C. Wyant

Roland V. Shack

Radiometry and

Optical Systems

William L. Wolfe

Philip N. Slater

For further details, please write to:

\section{Optical Systems and Engineering Short Courses, Inc. \\ P.O.Box 18667}

Tucson, Arizona 85731

Or telephone

Philip N. Slater, the short course director, at $602885-3798$. 